\title{
Holographic Screens in Ultraviolet Self-Complete Quantum Gravity
}

\author{
Piero Nicolini ${ }^{1,2}$ and Euro Spallucci ${ }^{3}$ \\ ${ }^{1}$ Frankfurt Institute for Advanced Studies (FIAS), Ruth-Moufang-Straße 1, 60438 Frankfurt am Main, Germany \\ ${ }^{2}$ Institut für Theoretische Physik, Johann Wolfgang Goethe-Universität, Max-von-Laue-Straße 1, \\ 60438 Frankfurt am Main, Germany \\ ${ }^{3}$ Dipartimento di Fisica, Sezione di Fisica Teorica, Università degli Studi di Trieste e INFN, Sezione di Trieste, \\ Strada Costiera 11, 34151 Trieste, Italy \\ Correspondence should be addressed to Piero Nicolini; nicolini@th.physik.uni-frankfurt.de
}

Received 29 January 2014; Accepted 3 March 2014; Published 27 March 2014

Academic Editor: Christian Corda

Copyright (C) 2014 P. Nicolini and E. Spallucci. This is an open access article distributed under the Creative Commons Attribution License, which permits unrestricted use, distribution, and reproduction in any medium, provided the original work is properly cited. The publication of this article was funded by SCOAP ${ }^{3}$.

\begin{abstract}
This paper studies the geometry and the thermodynamics of a holographic screen in the framework of the ultraviolet self-complete quantum gravity. To achieve this goal we construct a new static, neutral, nonrotating black hole metric, whose outer (event) horizon coincides with the surface of the screen. The spacetime admits an extremal configuration corresponding to the minimal holographic screen and having both mass and radius equalling the Planck units. We identify this object as the spacetime fundamental building block, whose interior is physically unaccessible and cannot be probed even during the Hawking evaporation terminal phase. In agreement with the holographic principle, relevant processes take place on the screen surface. The area quantization leads to a discrete mass spectrum. An analysis of the entropy shows that the minimal holographic screen can store only one byte of information, while in the thermodynamic limit the area law is corrected by a logarithmic term.
\end{abstract}

\section{Introduction}

"Quantum gravity" is the common tag for any attempt to reconcile gravity and quantum mechanics. Since the early proposals by Wheeler [1,2] and DeWitt [3], up to the recent ultraviolet (UV) self-complete scenario [4], the diverse formulations of a would-be quantum theory of gravity have shown a common feature, that is, a fundamental length/energy scale where the smooth manifold model of spacetime breaks down. Let us refer to this scale as the "Planck scale" irrespectively whether it is $10^{19} \mathrm{GeV}$ or $10-10^{2} \mathrm{TeV}$. The very concept of distance becomes physically meaningless at the Planck scale and spacetime "evaporates" into something different, a sort of "foamy" structure, a spin network, a fractal dust, and so forth, according to the chosen model [5]. As a matter of fact, one of the most powerful frameworks for describing the Planckian phase of gravity is definitely (Super) String Theory. The price to pay to have a perturbatively finite, anomaly-free quantum theory is to give up the very idea of point-like building blocks of matter and replace them with one-dimensional vibrating strings. As there does not exist any physical object smaller than a string; there are no physical ways to probe distances smaller than the length of the string itself. In this regard two properties of fundamental strings are worth mentioning:

(i) string excitations correspond to different mass and spin "particle" states;

(ii) highly excited strings share various physical properties with black holes.

Thus, we infer that string theory provides a bridge between particle-like objects and black holes (see for instance [6]). However, it is important to remark that while the Compton wavelength of a particle-type excitation decreases by increasing the mass, the Schwarzschild radius of a black hole increases with its mass. Thus, the first tenet of high energy 
particle physics, which is "the higher the energy the shorter the distance," breaks down when gravity comes into play and turns a "particle" into a black hole. The above remark is the foundation of the UV self-complete quantum gravity scenario, where the Planckian and sub-Planckian length scales are permanently shielded from observation due to the production of black hole excitations at Planck energy scattering [7]. Accordingly the Planck scale assumes the additional meaning of scale at which matter undergoes a transition between its two admissible "phases," that is, the particle phase and the black hole phase [8-10]. From this perspective, transPlanckian physics is dominated by larger and larger black hole configurations. It follows that only black holes larger than, or at most equal to, Planck size objects can self-consistently fit into this scheme. However, classical black hole solutions do not fulfill this requirement, that is, the existence of a lower bound for their mass and size (see Figure 1).

A first attempt to overcome this limitation is offered by the noncommutative geometry inspired solutions of the Einstein equations [11]. The latter are a family of regular black holes which span all possible combinations of parameters, such as mass [12], charge [13], and angular momentum $[14,15]$. In addition such regular geometries admit a variety of complementary gravitational configurations such as traversable wormholes [16], dirty black holes [17], dilaton gravity black holes [18], and collapsing matter shells [19]. Recently this family of black holes has been recognized as viable solutions of nonlocal gravity [20,21], that is, a set of theories exhibiting an infinite number of derivative terms of the curvature scalar [22-24] in place of the mere Ricci scalar as in the standard Einstein-Hilbert action. More importantly extensions of noncommutative geometry inspired metrics to the higher dimensional scenario $[25,26]$ are currently under scrutiny at the LHC for their unconventional phenomenology [27]: specifically the terascale black holes described by such regular metrics tend to have a slower evaporation rate [28] and emit only soft particles mainly on the four-dimensional brane [29]. A characteristic feature of this type of solutions is that the minimum size configuration is given by the extremal black hole configuration which exists even in the neutral nonspinning case [30-32]. This fact automatically implies a minimum energy for black hole production in particle collisions [33] without any further need of correcting formulas of cross sections with ad hoc threshold functions. Extremal configurations play a crucial role in the physics of the decaying de Sitter universe via the nucleation of microscopic black holes. It has been shown that Planck size noncommutative inspired black holes might have been copiously produced during inflationary epochs [34]. This fact has further phenomenological repercussions: being stable, noninteracting objects, extremal black holes turn out to be a reliable candidate for dark matter component. On the theoretical side, extremal configurations in the presence of a negative cosmological term can provide a short scale completion of the Hawking-Page diagram which switches to a more realistic Van der Waals phase diagram [35].

Extremal configurations can be either descending from the introduction of a fundamental length in the line element and can alternatively be interpreted as a phenomenological

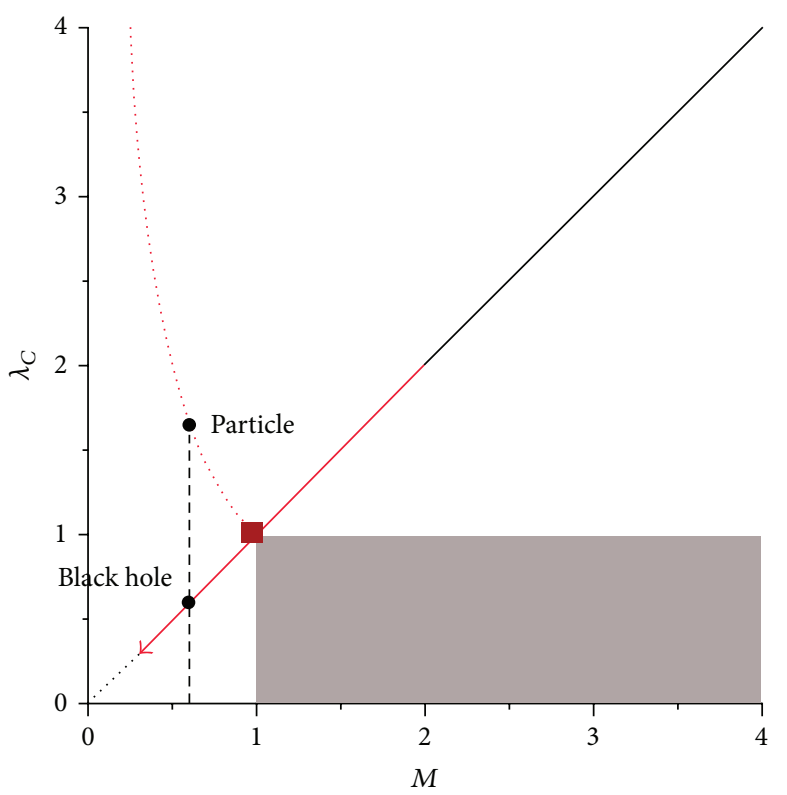

Figure 1: The dotted and the solid curves represent the particle Compton wavelength $\lambda_{C}$ and the Schwarzschild radius as a function of the energy $M$ in Planck units (quantities are rescaled). The squared bullet is the Planck scale. The grey area of the diagram is actually excluded, meaning that a particle cannot be compressed at distances smaller than the Planck length: at trans-Planckian energy only black hole form. The arrow shows the inadequacy of the Schwarzschild metric: black holes have no lower mass bounds, can have size smaller than the Planck length, and can expose the curvature singularity by decaying through the Hawking process.

input from quantum gravity: in the latter case it has been shown that such extremal black holes fit pretty well in the UV self-complete scenario providing a stable, minimum size probe at the transition point between particles and black holes [36].

In this paper we want to take a step further in the realization of this program by avoiding the introduction of an additional principle to justify the presence of a minimal length, rather we demand the radius of a Planck size extremal black hole to provide the natural UV cutoff of a quantum spacetime. In this framework gravity is expected to be selfregular in the sense that the actual regulator cutting off subPlanckian length scales is given in terms of the gravitational coupling constant; that is, $\sqrt{G}=L_{P}$. The paper is organized as follows. In Section 2 we derive a black hole metric, consistent with the above discussion and the concept of holographic screen. The latter coincides with the outer horizon of the black hole whose mass spectrum is bounded from below by the mass of the extremal configuration equalling the Planck mass. Once trans-Planckian length scales are cut-ff, the "interior" of the black hole loses its physical meaning in the sense that all the relevant degrees of freedom are necessarily located on the horizon itself. In Section 3 we discuss the thermodynamics of the screen. We find that the area law is modified by logarithmic corrections and that there exists a minimal holographic screen with zero thermodynamic 
entropy. Finally we propose a "holographic quantization" scheme where the area of the extremal configuration provides the quantum of surface. In Section 4 we offer the reader a brief summary of the main results of this work.

\section{Self-Regular Holographic Screen}

A simple but intriguing model of singularity-free black hole has been "guessed" in [37], in the sense that the metric was assigned as an input for the Einstein equations. Sometimes this inverted procedure is called "engineering" because the actual source term of field equations is not known a priori. The distinctive feature of the solution is the presence in the line element of a free parameter with dimension of a length, acting as a short distance regulator for the spacetime curvature, allowing a safe investigation of back-reaction effects of the Hawking radiation. In [38] a higher dimensional extension of this model has been proposed; it was also shown that, by a numerical rescaling of the short-distance regulator, it is possible to identify this fundamental length scale with the radius of the extremal configuration. With hindsight, we are going to take a step forward to improve this inverse procedure. Specifically, we want to follow the "direct way" by building up a consistent source for Einstein equations: we introduce a physically motivated energy momentum tensor which allows for transitions between particle-like objects and black holes as consistently required by UV self-complete quantum gravity.

We start from the energy density for a point-particle in spherical coordinates as

$$
\rho_{p}(r)=\frac{M}{4 \pi r^{2}} \delta(r),
$$

where $\delta(r)$ is the Dirac delta. The energy distribution (1) implies a black hole for any value of mass $M$ even for subPlanckian values where one expects just particles. Before proceeding, we would like to recall that a Dirac delta function can be represented as the derivative of a Heaviside stepfunction $\Theta$ :

$$
\delta(r)=\frac{d}{d r} \Theta(r) .
$$

Against this background, we want to accommodate both particles and black holes by a suitable modification of the energy distribution in order to overcome the ambiguities of the Schwarzschild metric in the sub-/trans-Planckian regimes (see also Figure 1). This can be done by considering a "smooth" function $h(r)$ in place of the Heaviside step:

$$
\Theta(r) \longrightarrow h(r) \text {. }
$$

The new profile $\rho(r)$ of the energy density is defined through $h(r)$ by the relation

$$
\rho(r)=\frac{M}{4 \pi r^{2}} \frac{d}{d r} h(r) \equiv T_{0}^{0} .
$$

By means of the conservation equation $\nabla_{\mu} T^{\mu \nu}=0$ one can determine the remaining components of the stress tensor, which turns to be out of the form

$$
T_{\mu}^{v}=\operatorname{diag}\left(-\rho, p_{r}, p_{\perp}, p_{\perp}\right) .
$$

The condition for the metric coefficients $g_{00}=-g_{11}^{-1}$ determines the equation of state, namely, the relation between the energy density and the radial pressure, $p_{r}=-\rho$. The angular pressure is specified by the conservation of the stress tensor and reads $p_{\perp}=p_{r}+(r / 2) \partial_{r} p_{r}$.

By plugging the tensor (5) in Einstein equations, one finds that the metric reads $(G=1)$

$$
\begin{aligned}
d s^{2}= & -\left(1-\frac{2 m(r)}{r}\right) d t^{2} \\
& +\left(1-\frac{2 m(r)}{r}\right)^{-1} d r^{2}+r^{2} d \Omega^{2},
\end{aligned}
$$

with

$$
m(r)=4 \pi \int d r^{\prime}\left(r^{\prime}\right)^{2} \rho\left(r^{\prime}\right)
$$

At large distances $r \gg L_{P}$, the above energy density has to quickly vanish; that is, $\rho(r) \rightarrow 0$ in order to match the "vacuum" Schwarzschild metric. Conversely, at shorter scales $r \gtrsim L_{P}$, the density $\rho(r)$ (and accordingly $h(r)$ ) has to depart from the point-particle profile in order to fulfill the following requirements:

(i) no curvature singularity in the origin;

(ii) self-implementation of a characteristic scale $l_{0}$ in the spacetime geometry by means of the radius of the extremal configuration $r_{0}$; that is, $r_{0}=l_{0}$.

The latter condition is crucial. For instance noncommutative geometry inspired black holes [11] are derived by the direct way; they enjoy (i) but fail to fulfill the condition (ii). This means that the characteristic length scale of the system $l_{0}$ and the extremal configuration radius $r_{0}$ are independent quantities. Indeed noncommutative geometry is the underlying theory which provides the scale $l_{0}$ in terms of an "external" parameter, namely, the noncommutative parameter $\theta$. In other words one needs to invoke a principle, like a modification of commutators in quantum mechanics, or the emergence of a quantum gravity induced fundamental length to achieve the regularity of the geometry at short scales. Against this background, we want just to use $r_{0}$ as fundamental scale, getting rid of any $l_{0}$ as emerging from any theory or principle not included in Einstein field equations. This is a step forward since it opens the possibility for Einstein gravity to be self-protected in the ultraviolet regime. To emphasize this point, we introduced the word "selfimplementation" in (ii). Since there exists actually only one additional scale beyond $r_{0}$, that is, the Planck length $L_{P}=$ $\sqrt{G}$, or the Planck mass $M_{P}=1 / \sqrt{G}$, we can implement the condition (ii) in the most natural way by setting $r_{0}=L_{P}$ and accordingly $M_{0}=M_{P}$, where $M_{0} \equiv M\left(r_{0}\right)$ is the extremal black hole mass.

Despite the virtues of the above line of reasoning, we feel that the set of conditions (i) and (ii) can be relaxed and a further simplification is possible. Having in mind that for extremal black hole configurations the Hawking emission stops we just need to find a metric for which only the condition (ii) holds. This would be enough for completing 
the program of the UV self-complete quantum gravity by protecting the short distance behavior of gravity during the final stages of the evaporation process. In this regard, the resulting extremal black hole is just the smallest object one can use to probe short-distance physics. In other words, in the framework of UV self-complete quantum gravity, it is not physically meaningful to ask about curvature singularity inside the horizon as the very concept of spacetime is no longer defined below this length scale.

According with such a line of reasoning, we can determine the function $h(r)$ by dropping the condition (i) and keeping just the condition (ii). Inside the class of all admissible profiles for $h(r)$, the most natural and algebraically compact choice is given by

$$
h(r)=1-\frac{L_{P}^{2}}{r^{2}+L_{P}^{2}} .
$$

A similar procedure has been already used in [33] and accounts for the fact that in the presence of $L_{P}$ the step cannot be any longer sharp. Thus, the smeared energy density $\rho(r)$ turns out to be

$$
\rho(r)=\frac{M}{2 \pi r} \frac{L_{P}^{2}}{\left(r^{2}+L_{P}^{2}\right)^{2}} .
$$

As a result we find the following metric which is derived from a stress tensor modeling a particle-black hole system (5):

$$
\begin{aligned}
d s^{2}= & -\left(1-\frac{2 M L_{P}^{2} r}{r^{2}+L_{P}^{2}}\right) d t^{2} \\
& +\left(1-\frac{2 M L_{P}^{2} r}{r^{2}+L_{P}^{2}}\right)^{-1} d r^{2}+r^{2} d \Omega^{2},
\end{aligned}
$$

where the arbitrary constant $M$ is defined as follows:

$$
M \equiv \frac{1}{2 L_{P}^{2} r_{h}}\left(r_{h}^{2}+L_{P}^{2}\right) .
$$

We give $M$ the physical meaning of mass for a spherical, holographic screen with radius $r_{h}$. The basic idea is that gravitational phenomena taking place in three-dimensional space can be projected on a two-dimensional "viewing screen" with no loss of information [39]. The idea of holographic screen has been proposed in [40] and it has mathematically been formulated in [41]: the holographic screen plays the role of "basic constituent of space where the Newton potential is constant." Along this line of reasoning, the idea of holographic screen has been used also in the context of noncommutative inspired metric to derive compelling deviations to Newton's law [42]. For what concerns the current discussion, however, we just need to recall that a special case of holographic screen is given by an event horizon where the entropy is maximized.

Several remarks are in order.

(i) It is easy to show that $M \geq M_{P}$ and equals the Planck mass only for $r_{h}=L_{P}$. (ii) The line element (10) admits a pair of horizons provided $M \geq M_{P}$. The radii $r_{ \pm}$of the horizons are given by

$$
r_{ \pm}=L_{P}^{2}\left(M \pm \sqrt{M^{2}-M_{P}^{2}}\right) .
$$

For $M=M_{P}$ the two horizons merge into a single (degenerate) null surface at $r_{ \pm}=r_{0}=L_{P}$. For $M \gg$ $M_{P}$ the outer horizon approaches the conventional value of the Schwarzschild geometry; that is, $r_{+} \simeq$ $2 M L_{P}^{2}$.

(iii) By inserting (11) into (12) one finds $r_{+}=r_{h}, r_{-}=$ $L_{P}^{2} / r_{h}$. We see that the holographic screen surface coincides with the (outer) black hole horizon $r_{+}$, while the inner Cauchy horizon has a radius which is always smaller or equal to the Planck length. This fact lets us circumvent the issue of potential blue shift instabilities [43, 44] (see, i.e., recent analyses for noncommutative inspired $[45,46]$ and other quantum gravity corrected metrics $[47,48]$ ) because $r_{-}$simply loses its physical meaning being not accessible to any sort of measurement process. In what follows we can identify the holographic screen with the black hole outer horizon without distinguishing between the two surfaces any longer.

(iv) "Light" objects, with $M<M_{P}$, are "particles" rather than holographic screens. By particles we mean localized lumps of energy of linear size given by the Compton wavelength $\lambda_{C}=1 / M$ that can never collapse into a black hole. Rather they give rise to horizonless metrics (see Figure 2) and cannot probe distances smaller than $\lambda_{C}$. The "transition" particle $\rightarrow$ black holes is discussed below in terms of critical surface density.

As a further analysis of this result, it is interesting to consider the surface energy density of the holographic screen which is defined as

$$
\sigma_{h} \equiv \frac{M}{4 \pi r_{h}^{2}}=\frac{1}{8 \pi L_{P}^{2}} \frac{r_{+}^{2}+L_{P}^{2}}{r_{+}^{3}} .
$$

From the above relation we see that $\sigma_{h}$ is a monotonically decreasing function of the screen radius. We notice that there exists a minimal screen encoding the physically maximum attainable energy density, that is, the Planck (surface) density:

$$
\sigma_{h}\left(r_{+}=L_{P}\right)=\frac{1}{4 \pi L_{P}^{3}}=\frac{M_{P}}{4 \pi L_{P}^{2}} .
$$

We stress that there is no physically meaningful "interior" for the minimal screen; that is, the "volume" of such an object is not even defined, in the sense that it can never be probed. Thus, we can only consider energy per unit area, rather than per unit volume. If we, formally, define a surface energy for a particle as

$$
\sigma_{p} \equiv \frac{M}{4 \pi \lambda_{C}^{2}}=\frac{1}{4 \pi \lambda_{C}^{3}}
$$


we see that the two curves (13) and (15) cross at $\lambda_{C}=$ $L_{P}=r_{+}$. This result offers an additional interpretation for the Planck length which consistently turns to be the minimal size for a particle as well for a black hole (see Figure 2). Accordingly, the Planck density (14) is the critical density for a particle to collapse into a black hole. This argument is usually formulated in terms of volume energy density having in mind the picture of macroscopic body gravitationally collapsing under their own weight. From our holographic vantage point, where "surfaces" are the basic dynamical objects, it is natural to reformulate this reasoning in terms of areal densities [39]. In addition holography offers a way to circumvent potential conflicts between the mechanism of spontaneous dimensional reduction $[49,50]$ and the UV self-complete paradigm. If we perform the limit for $r \rightarrow 0$ the metric (10) would apparently reduce into an effective two-dimensional spacetime:

$$
d s^{2} \longrightarrow-(1-2 M r) d t^{2}+(1-2 M r)^{-1} d r^{2}+\mathcal{O}\left(\frac{r^{2}}{L_{P}^{2}}\right) .
$$

As explained in [51], this mechanism would lead the formation of lower dimensional black holes for length scales below the Planck length, in contrast with the predicted semiclassical regime of trans-Planckian black holes in four dimensions. However, contrary to the Schwarzschild metric that eventually reduces into dilaton gravity black holes when $r \simeq L_{P}$ (for reviews of the mechanism see $[52,53]$ ), the presence of the holographic screen forbids the access to length scales $r<L_{P}$ and safely protects the arguments at the basis of the UV self-complete quantum gravity.

\section{Thermodynamics, Area Quantization, and Mass Spectrum}

In this section we would like to investigate the thermodynamics of the black hole described by (10) and determine the relation between entropy and area of the event horizon. It is customary to consider the area law for granted in any case, but this assumption leads to an inconsistency with the third law of thermodynamics: extremal black holes have zero temperature but nonvanishing area. Here, we stick to the textbook definition of thermodynamical entropy and not to more exotic quantity like Rényi, or entanglement entropy. To cure this flaw, we will derive the relation between entropy and area from the first law, rather than assuming it. The Hawking temperature associated to the metric (10) can be calculated by evaluating the surface gravity $\kappa$ :

$$
T_{H}=\frac{\kappa}{2 \pi}=\frac{1}{4 \pi}\left(\frac{d g_{00}}{d r}\right)_{r=r_{+}}=\frac{1}{4 \pi r_{+}}\left(1-\frac{2 L_{P}^{2}}{r_{+}^{2}+L_{P}^{2}}\right)
$$

while the heat capacity $C \equiv \partial U / \partial T_{H}$ is

$$
C \equiv \frac{\partial M}{\partial T_{H}}=-2 \pi r_{+}\left(\frac{r_{+}^{2}-L_{P}^{2}}{L_{P}^{2}}\right) \frac{\left(r_{+}^{2}+L_{P}^{2}\right)^{2}}{r_{+}^{4}-4 L_{P}^{2} r_{+}^{2}-L_{P}^{4}} .
$$

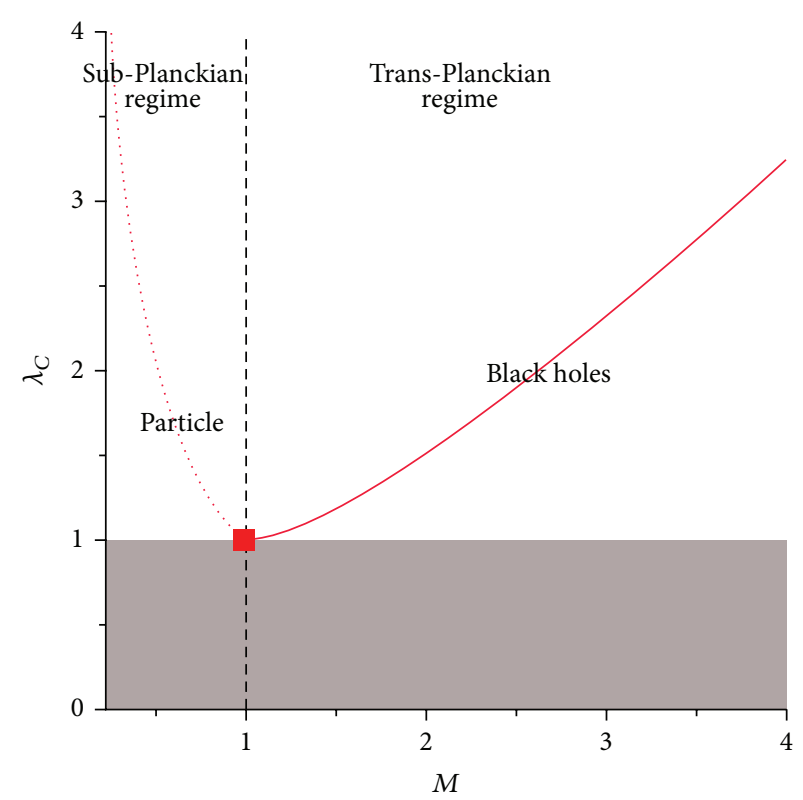

FIGURE 2: The plot shows a length/energy relation consistent with the self-complete quantum gravity arguments in Planck units. Particles (dotted line) and black holes (solid line) cannot probe length shorter than the Planck length. The grey area is permanently inaccessible and accordingly represents the minimal spacetime time region or fundamental constituent, that is, the "atom" the spacetime is supposed to be made of.

One can check that for large distances, that is, $r_{+} \gg L_{P}$, both (17) and (18) coincide with the conventional results of the Schwarzschild metric; that is, $T_{H} \approx 1 / 4 \pi r_{+}$and $C \approx-2 \pi r_{+}^{2}$ (see Figures 3 and 4 ). On the other hand at Planckian scales, contrary to the standard result for which a Planckian black hole has a temperature $T_{H}=M_{P} / 8 \pi$, we have that $T_{H} \rightarrow 0$ as $r_{+} \rightarrow r_{0}=L_{P}$ as expected for any extremal configurations. This discrepancy with the classical picture is consistent with the genuine quantum gravitational character of the black hole and is reminiscent of the modified thermodynamics of noncommutative inspired black holes $[54,55]$.

The Hawking emission is a semiclassical decay where gravity is considered just in terms of a classical spacetime background. Such a semiclassical approximation conventionally breaks down as the Planck scale is approached. On the other hand for our metric, at $r_{+}=r_{M}=\sqrt{2+\sqrt{5}} L_{P} \simeq$ $2.058 L_{P}$ the temperature admits a maximum corresponding to a pole in the heat capacity. In the final stage of the evaporation, that is, $L_{P}<r_{+}<r_{M}$, the heat capacity is positive; the Hawking emission slows down and switches off at $r_{+}=L_{P}$. From a numerical estimate of the maximum temperature one finds $T_{H}\left(r_{M}\right)=0.0239 M_{P}$. This implies that the ratio temperature/mass is $T_{H} / M<T_{H}\left(r_{M}\right) / M_{0} \simeq 0.0239$. As a consequence, no relevant back reaction occurs during all the evaporation processes and the metric can consistently describe the system "black hole + radiation" for all $r_{+} \geq L_{P}$.

We can summarize the process with the following scheme: 


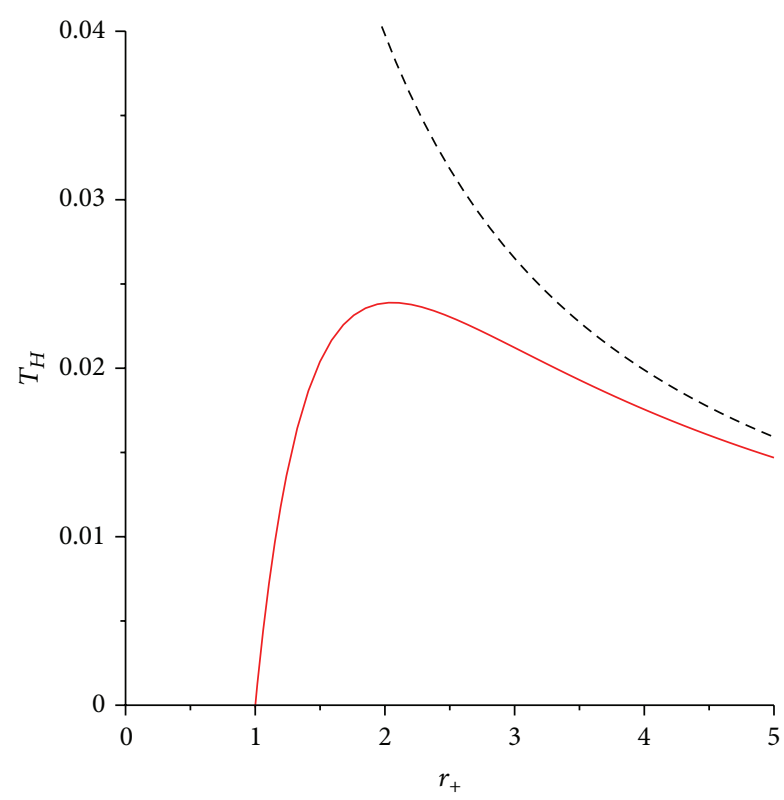

Figure 3: The solid curve represents the Hawking temperature $T_{H}$ and as a function of the horizon radius $r_{+}$in Planck units. The dotted curve represents the corresponding classical result in terms of the Schwarzschild metric.

(i) "large", far-from-extremality, black holes are semiclassical objects which radiate thermally;

(ii) "small", quasi-extremal, black holes are quantum objects;

(iii) $r=r_{M}$ is "critical point" where the heat capacity diverges (see Figure 4). Since $C>0$ for $r_{0}<r_{+}<r_{M}$ and $C<0$ for $r_{M}<r_{+}$, we conclude that a phase transition takes place from large thermodynamically unstable black holes to small stable black holes.

As a matter of fact, the black hole emission preceding the evaporation switching off (often called "SCRAM phase" [11]) might not be thermal. It has been argued that such a quantum regime might be characterized by discrete jumps towards the ground state $[7,56]$. To clarify the nature of this mechanism we proceed by studying the black hole entropy profile and the related area quantization. By integrating the first law, taking into account that no black hole can have a radius smaller than $r_{0}=L_{P}$, that is,

$$
S\left(r_{+}\right)=\int_{r_{0}}^{r_{+}} \frac{d M}{T_{H}}=\frac{\pi}{L_{P}^{2}}\left(r_{+}^{2}-L_{P}^{2}\right)+2 \pi \ln \left(\frac{r_{+}}{L_{P}}\right),
$$

we can cast the entropy in terms of the area of the event horizon $\mathscr{A}_{+} \equiv 4 \pi r_{+}^{2}$ as

$$
S\left(\mathscr{A}_{+}\right)=\frac{\pi}{\mathscr{A}_{0}}\left(\mathscr{A}_{+}-\mathscr{A}_{0}\right)+\pi \ln \left(\frac{\mathscr{A}_{+}}{\mathscr{A}_{0}}\right)
$$

where $\mathscr{A}_{0}=4 \pi L_{P}^{2}$ is the area of the extremal event horizon. We remark that the modifications to the Schwarzschild metric, encoded in our model, are in agreement with all the major approaches to quantum gravity, which universally foresee a logarithmic term as a correction to the classical area law. For brevity we recall that this is the case for string theory $[57,58]$, loop quantum gravity [59-61], and other results based on generic arguments [62, 63], on Cardy's formula [64], conformal properties of spacetimes [65], and other mechanisms for counting microstates [66-68]. We can check that this is the case for the metric (10) by performing the limit $r_{+} \gg L_{P}$ for (20) to obtain

$$
S\left(\mathscr{A}_{+}\right) \approx \frac{\mathscr{A}_{+}}{4 L_{P}^{2}}+\pi \ln \left(\frac{\mathscr{A}_{+}}{4 \pi L_{P}^{2}}\right) .
$$

Conversely for $r_{+} \rightarrow L_{P}$ the entropy vanishes; that is,

$$
S\left(\mathscr{A}_{+}\right) \approx \frac{4 \pi}{L_{P}}\left(r_{+}-L_{P}\right)+O\left(\left(r_{+}-L_{P}\right)^{2}\right) .
$$

This result is consistent both with the third law of thermodynamics and the entropy statistical meaning. The Planck size, zero temperature, black hole configuration is the unique ground state for holographic screens. Thus, it is a zero entropy state as there is only one way to realize this configuration. To see this we promote the extremal configuration area to the fundamental quantum of area:

$$
\mathscr{A}_{+} \equiv \mathscr{A}_{n-1}=n \mathscr{A}_{0}=4 \pi n L_{P}^{2},
$$

where $L_{P}^{2}$ represents the basic information pixel and $n=$ $1,2,3 \ldots$ is the number of bytes (we borrow here the names of some units of digital information. In the present context, each byte consists of $4 \pi$ bits. Each bit, represented by $L_{P}^{2}$ is the basic capacity of information of the holographic screen. In the analogy with the theory of information for which a byte represents the minimum amount of bits for encoding a single character of text, here the byte represents the minimum number of basic pixel $L_{P}^{2}$ for encoding the smallest holographic screen). From the above condition one obtains

$$
\begin{aligned}
& r_{n-1} \equiv n^{1 / 2} L_{P}, \\
& M_{n-1} \equiv \frac{1}{2}\left(n^{1 / 2}+n^{-1 / 2}\right) M_{P} .
\end{aligned}
$$

Consistently the ground state of the system is $r_{0}=L_{P}$ and $M_{0}=M_{P}$, while for $n \gg 1$ one finds a continuous spectrum of values. This can be checked through the following relation:

$$
\Delta M_{n} \equiv M_{n}-M_{n-1} \sim \frac{1}{4} n^{-1 / 2} M_{P} .
$$

We notice that for $n \leq 4$ we are in the regime of positive heat capacity $C>0$ and discrete mass spectrum, while for $n>4$ we approach the semiclassical limit characterized by negative heat capacity $C<0$ and continuous mass spectrum; that is, $\Delta M_{n} / M_{n} \leq 1 / 12$. This confirms that at $r_{+}=r_{M}$, the system undergoes a phase transition from a semiclassical regime to a genuine quantum gravity regime. As a conclusion we have that large black holes decay thermally, while small objects decay quantum mechanically, by emitting quanta of 


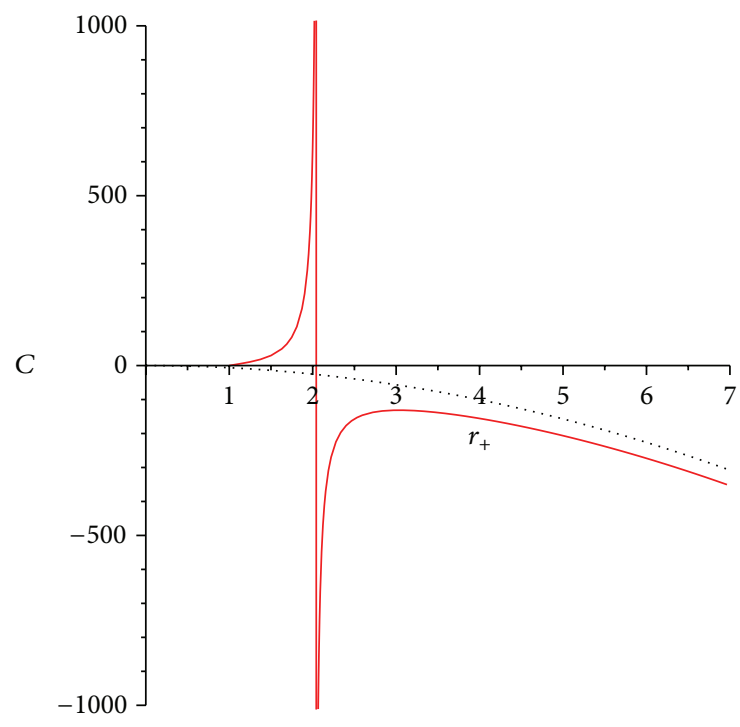

Figure 4: The solid curve represents the black hole heat capacity $C$ as a function of the horizon radius $r_{+}$in Planck units. The dotted curve represents the corresponding classical results in terms of the Schwarzschild metric.

energy (for a recent phenomenological analysis of such kind of decay see [69]). The end point of the decay is a Planck mass, holographic screen.

The quantization of the area of the holographic screen lets us disclose further features of the informational content of the holographic screen. We have that the surface density can be written as

$$
\sigma_{h}(n)=\frac{1}{2}\left(\frac{1}{n^{1 / 2}}+\frac{1}{n^{3 / 2}}\right) \frac{M_{P}}{4 \pi L_{P}^{2}},
$$

while the entropy reads $S(n)=\pi(n+\ln (n)-1)$. From this relation we learn that, while the entropy increases with the number $n$ of bytes, the surface density decreases. This confirms that the extremal configuration is nothing but a single byte, zero entropy, Planckian density holographic screen.

\section{Discussion and Conclusions}

In this paper we have presented a neutral nonspinning black hole geometry admitting an extremal configuration whose mass and radius coincide with the Planck units. We have reached this goal by suitably modelling a stress tensor able to accommodate both the particle and black hole configurations, undergoing a transition at the Planck scale. We showed that the horizon of the degenerate black hole represents the minimal holographic screen, within which we cannot access any information about the matter-energy content of spacetime.

We showed that a generic holographic screen is described in terms of the outer horizon of the metric (10), while the inner horizon lies within the prohibited region, that is, inside the minimal holographic screen. The whole scheme fits into the gravity self-completeness scenario. For sub-Planckian energy scales one has just a quantum particle able to probe at the most distances of the order of its Compton wavelength. By increasing the degree of compression of the particle, one traverses the Planck scale where a collapse into a black hole occurs, before probing a semiclassical regime at transPlanckian energies. The virtual curvature singularity of the geometry in $r=0$ is therefore wiped out since in such a context sub-Planckian lengths have no physical meaning. From this vantage point spacetime stops to exist beyond the Planck scale as there is no physical way to access this regime. Thus, the curvature singularity problem is ultimately resolved by giving up the very concept of spacetime at sub-Planckian length scales.

The study of the associated thermodynamic quantities confirmed that at trans-Planckian energies black holes radiate thermally before undergoing a phase transition to smaller, quantum black holes. The latter decay by emitting a discrete spectrum of quanta of energy and reach the ground state of the evaporation corresponding to the minimal holographic screen. We came to this conclusion by quantizing the black hole horizon area in terms of the minimal holographic screen which actually plays the role of a basic information byte. We showed that in the thermodynamic limit, the area law for the black hole entropy acquires a logarithmic correction in agreement with all the major quantum gravity formulations.

In conclusion, we stress that the line element (10) not only captures the basic features of more "sophisticated" models of quantum gravity improved black holes (e.g., noncommutative geometry inspired black holes [11], loop quantum gravity black holes $[70,71]$, asymptotically safe gravity black holes $[72,73]$, and other studies about collapses in quantum gravity $[74,75])$ but overcomes some of their current weak points: specifically there is no longer any concern for potential Cauchy instabilities or for conflicts between the gravity selfcompleteness and the Planck scale spontaneous dimensional reduction mechanism, as well as the scenario of the terminal phase of the evaporation for static, nonrotating, neutral black holes. In addition, for its compact form the new metric allows straightforward analytic calculations and opens the route to testable predictions.

\section{Conflict of Interests}

The authors declare that there is no conflict of interests regarding the publication of this paper.

\section{References}

[1] J. A. Wheeler, "On the nature of quantum geometrodynamics," Annals of Physics, vol. 2, no. 6, pp. 604-614, 1957.

[2] J. A. Wheeler, "Physics at the planck length," International Journal of Modern Physics A, vol. 8, pp. 4013-4018, 1993.

[3] B. S. DeWitt, "Quantum theory of gravity. I. The canonical theory," Physical Review, vol. 160, p. 1113, 1967.

[4] G. Dvali and C. Gomez, "Self-completeness of Einsteingravity," http://arxiv.org/abs/1005.3497.

[5] L. J. Garay, "Quantum gravity and minimum length," International Journal of Modern Physics A, vol. 10, no. 2, p. 145, 1995. 
[6] G. T. Horowitz and J. Polchinski, "Correspondence principle for black holes and strings," Physical Review D: Particles and Fields, vol. 55, no. 10, pp. 6189-6197, 1997.

[7] G. Dvali, S. Folkerts, and C. Germani, "Physics of transPlanckian gravity," Physical Review D: Particles and Fields, vol. 84, Article ID 024039, 2011.

[8] G. Dvali, G. F. Giudice, C. Gomez, and A. Kehagias, "Vcompletion by classicalization," Journal of High Energy Physics, vol. 2011, no. 8, article 108, 2011.

[9] G. Dvali and D. Pirtskhalava, "Dynamics of unitarization by classicalization," Physics Letters B: Nuclear, Elementary Particle and High-Energy Physics, vol. 699, no. 1-2, pp. 78-86, 2011.

[10] G. Dvali and C. Gomez, "Ultra-high energy probes of classicalization," Journal of Cosmology and Astroparticle Physics, vol. 2012, no. 7, article 015, 2012.

[11] P. Nicolini, "Noncommutative black holes, the final appeal to quantum gravity: a review," International Journal of Modern Physics A, vol. 24, no. 7, p. 1229, 2009.

[12] P. Nicolini, "A model of radiating black hole in noncommutative geometry," Journal of Physics A: Mathematical and General, vol. 38, no. 39, pp. L631-L638, 2005.

[13] S. Ansoldi, P. Nicolini, A. Smailagic, and E. Spallucci, "Noncommutative geometry inspired charged black holes," Physics Letters B: Nuclear, Elementary Particle and High-Energy Physics, vol. 645, no. 2-3, pp. 261-266, 2007.

[14] A. Smailagic and E. Spallucci, "'Kerrr' black hole: the lord of the string," Physics Letters B: Nuclear, Elementary Particle and HighEnergy Physics, vol. 688, no. 1, pp. 82-87, 2010.

[15] L. Modesto and P. Nicolini, "Charged rotating noncommutative black holes," Physical Review D: Particles and Fields, vol. 82, Article ID 104035, 2010.

[16] R. Garattini and F. S. N. Lobo, "Self-sustained traversable wormholes in noncommutative geometry," Physics Letters B: Nuclear, Elementary Particle and High-Energy Physics, vol. 671, no. 1, pp. 146-152, 2009.

[17] P. Nicolini and E. Spallucci, "Noncommutative geometryinspired dirty black holes," Classical and Quantum Gravity, vol. 27, no. 1, Article ID 015010, 10 pages, 2010.

[18] J. R. Mureika and P. Nicolini, "Aspects of noncommutative (1+ 1)-dimensional black holes," Physical Review D: Particles and Fields, vol. 84, Article ID 044020, 2011.

[19] P. Nicolini, A. Orlandi, and E. Spallucci, "The final stage of gravitationally collapsed thick matter layers," Journal of High Energy Physics, vol. 2013, Article ID 812084, 8 pages, 2013.

[20] L. Modesto, J. W. Moffat, and P. Nicolini, "Black holes in an ultraviolet complete quantum gravity," Physics Letters B: Nuclear, Elementary Particle and High-Energy Physics, vol. 695, no. 1-4, pp. 397-400, 2011.

[21] P. Nicolini, "Nonlocal and generalized uncertainty principle blackholes," http://arxiv.org/abs/1202.2102.

[22] T. Biswas, E. Gerwick, T. Koivisto, and A. Mazumdar, “Towards singularity- and ghost-free theories of gravity," Physical Review Letters, vol. 108, no. 3, Article ID 031101, 2012.

[23] L. Modesto, "Super-renormalizable quantum gravity," Physical Review D: Particles and Fields, vol. 86, Article ID 044005, 2012.

[24] L. Modesto, "Super-renormalizable multidimensional quantum gravity," New Astronomy Reviews, vol. 8, no. 2, pp. 4-33, 2013.

[25] T. G. Rizzo, "Noncommutative inspired black holes in extra dimensions," Journal of High Energy Physics, vol. 2006, no. 9, article 021, 2006.
[26] E. Spallucci, A. Smailagic, and P. Nicolini, "Non-commutative geometry inspired higher-dimensional charged black holes," Physics Letters B: Nuclear, Elementary Particle and High-Energy Physics, vol. 670, no. 4-5, pp. 449-454, 2009.

[27] D. M. Gingrich, "Noncommutative geometry inspired black holes in higher dimensions at the LHC," Journal of High Energy Physics, vol. 2010, no. 5, article 022, 2010.

[28] R. Casadio and P. Nicolini, "The decay-time of noncommutative micro-black holes," Journal of High Energy Physics, vol. 2008, no. 11, article 072, 2008.

[29] P. Nicolini and E. Winstanley, "Hawking emission from quantum gravity black holes," Journal of High Energy Physics, vol. 2011, no. 11, article 075, 2011.

[30] P. Nicolini, A. Smailagic, and E. Spallucci, "Noncommutative geometry inspired Schwarzschild black hole," Physics Letters B: Nuclear, Elementary Particle and High-Energy Physics, vol. 632, no. 4, pp. 547-551, 2006.

[31] I. Arraut, D. Batic, and M. Nowakowski, "Maximal extension of the Schwarzschild space-time inspired by noncommutative geometry," Journal of Mathematical Physics, vol. 51, no. 2, Article ID 022503, 11 pages, 2010.

[32] R. Banerjee, S. Gangopadhyay, and S. K. Modak, "Voros product, noncommutative Schwarzschild black hole and corrected area law," Physics Letters B: Nuclear, Elementary Particle and High-Energy Physics, vol. 686, no. 2-3, pp. 181-187, 2010.

[33] J. Mureika, P. Nicolini, and E. Spallucci, "Could any black holes be produced at the LHC?" Physical Review D: Particles and Fields, vol. 85, Article ID 106007, 2012.

[34] R. B. Mann and P. Nicolini, "Cosmological production of noncommutative black holes," Physical Review D: Particles and Fields, vol. 84, Article ID 064014, 2011.

[35] P. Nicolini and G. Torrieri, "The Hawking-Page crossover in noncommutative anti-deSitter space," Journal of High Energy Physics, vol. 2011, no. 8, article 097, 2011.

[36] E. Spallucci and S. Ansoldi, "Regular black holes in UV selfcomplete quantum gravity," Physics Letters B: Nuclear, Elementary Particle and High-Energy Physics, vol. 701, no. 4, pp. 471474, 2011.

[37] S. A. Hayward, "Formation and evaporation of regular black holes," Physical Review Letters, vol. 96, Article ID 031103, 2006.

[38] E. Spallucci and A. Smailagic, "Black holes production in selfcomplete quantum gravity," Physics Letters B: Nuclear, Elementary Particle and High-Energy Physics, vol. 709, no. 3, pp. 266269, 2012.

[39] L. Susskind, "The world as a hologram," Journal of Mathematical Physics, vol. 36, no. 11, pp. 6377-6396, 1995.

[40] E. P. Verlinde, "On the origin of gravity and the laws of Newton," Journal of High Energy Physics, vol. 2011, no. 4, article 029, 2011.

[41] Y.-X. Chen and J.-L. Li, "First law of thermodynamics on holographic screens in entropic force frame," Physics Letters B: Nuclear, Elementary Particle and High-Energy Physics, vol. 700, no. 5, pp. 380-384, 2011.

[42] P. Nicolini, "Entropic force, noncommutative gravity and ungravity," Physical Review D: Particles and Fields, vol. 82, Article ID 044030, 2010.

[43] E. Poisson and W. Israel, "Inner-horizon instability and mass inflation in black holes," Physical Review Letters, vol. 63, no. 16, pp. 1663-1666, 1989.

[44] E. Poisson and W. Israel, "Internal structure of black holes," Physical Review D: Particles and Fields, vol. 41, no. 6, pp. 1796$1809,1990$. 
[45] D. Batic and P. Nicolini, "Fuzziness at the horizon," Physics Letters B: Nuclear, Elementary Particle and High-Energy Physics, vol. 692, no. 1, pp. 32-35, 2010.

[46] E. Brown and R. Mann, "Instability of the noncommutative geometry inspired black hole," Physics Letters B: Nuclear, Elementary Particle and High-Energy Physics, vol. 694, no. 4-5, pp. 440-445, 2011.

[47] E. Brown, R. Mann, and L. Modesto, "Stability of self-dual black holes," Physics Letters B: Nuclear, Elementary Particle and HighEnergy Physics, vol. 695, no. 1-4, pp. 376-383, 2011.

[48] E. G. Brown, R. B. Mann, and L. Modesto, "Mass inflation in the loop black hole," Physical Review D: Particles and Fields, vol. 84, no. 10, Article ID 104041, 2011.

[49] G. 't Hooft, "Dimensional reduction in quantum gravity," in Salam Fest, World Scientific, Singapore, 1993, Essay dedicated to Abdus Salam.

[50] S. Carlip, "Spontaneous dimensional reduction in shortdistance quantum gravity?" in Proceedings of the 25th Max Born Symposium:, "The Planck Scale", Wroclaw, Poland, June 2009, Invited Talk.

[51] J. Mureika and P. Nicolini, "Self-completeness and spontaneous dimensional reduction," The European Physical Journal Plus, vol. 128, article 78, 2013.

[52] D. Grumiller, W. Kummer, and D. V. Vassilevich, "Dilaton gravity in two dimensions," Physics Reports, vol. 369, no. 4, pp. 327-430, 2002.

[53] D. Grumiller and R. Meyer, "Ramifications of lineland," Turkish Journal of Physics, vol. 30, no. 5, pp. 349-378, 2006.

[54] Y. S. Myung, Y.-W. Kim, and Y.-J. Park, "Thermodynamics and evaporation of the noncommutative black hole," Journal of High Energy Physics, vol. 2007, no. 2, article 012, 2007.

[55] R. Banerjee, B. R. Majhi, and S. Samanta, "Noncommutative black hole thermodynamics," Physical Review D: Particles, Fields, vol. 77, no. 12, Article ID 124035, 8 pages, 2008.

[56] P. Meade and L. Randall, "Black holes and quantum gravity at the LHC," Journal of High Energy Physics, vol. 2008, no. 5, article 003, 2008.

[57] A. Strominger and C. Vafa, "Microscopic origin of the Bekenstein-Hawking entropy," Physics Letters B: Nuclear, Elementary Particle and High-Energy Physics, vol. 379, no. 1-4, pp. 99-104, 1996.

[58] S. N. Solodukhin, "Entropy of the Schwarzschild black hole and the string-black-hole correspondence," Physical Review D: Particles and Fields, vol. 57, no. 4, pp. 2410-2414, 1998.

[59] C. Rovelli, "Black hole entropy from loop quantum gravity," Physical Review Letters, vol. 77, no. 16, pp. 3288-3291, 1996.

[60] A. Ashtekar, J. Baez, A. Corichi, and K. Krasnov, "Quantum geometry and black hole entropy," Physical Review Letters, vol. 80, no. 5, pp. 904-907, 1998.

[61] F. Caravelli and L. Modesto, "Holographic effective actions from black holes," Physics Letters B: Nuclear, Elementary Particle and High-Energy Physics, vol. 702, no. 4, pp. 307-311, 2011.

[62] S. N. Solodukhin, "Conical singularity and quantum corrections to the entropy of a black hole," Physical Review D: Particles and Fields, vol. 51, no. 2, pp. 609-617, 1995.

[63] R. B. Mann and S. N. Solodukhin, "Universality of quantum entropy for extreme black holes," Nuclear Physics B, vol. 523, no. 1-2, pp. 293-307, 1998.

[64] S. Carlip, "Logarithmic corrections to black hole entropy, from the Cardy formula," Classical and Quantum Gravity, vol. 17, no. 20, pp. 4175-4186, 2000.
[65] S. N. Solodukhin, "Conformal description of horizon's states," Physics Letters B: Nuclear, Elementary Particle and High-Energy Physics, vol. 454, no. 3-4, pp. 213-222, 1999.

[66] R. K. Kaul and P. Majumdar, "Quantum black hole entropy," Physics Letters B: Nuclear, Elementary Particle and High-Energy Physics, vol. 439, no. 3-4, pp. 267-270, 1998.

[67] R. K. Kaul and P. Majumdar, "Logarithmic correction to the Bekenstein-Hawking entropy," Physical Review Letters, vol. 84, no. 23, pp. 5255-5257, 2000.

[68] A. Sen, "Black hole entropy function, attractors and precision counting of microstates," General Relativity and Gravitation, vol. 40, no. 11, pp. 2249-2431, 2008.

[69] X. Calmet and N. Gausmann, "Nonthermal quantum black holes with quantized masses," International Journal of Modern Physics A. Particles and Fields. Gravitation. Cosmology, vol. 28, no. 13, Article ID 1350045, 21 pages, 2013.

[70] L. Modesto, "Loop quantum black hole," Classical and Quantum Gravity, vol. 23, no. 18, pp. 5587-5601, 2006.

[71] L. Modesto, "Black hole interior from loop quantum gravity," Advances in High Energy Physics, vol. 2008, Article ID 459290, 12 pages, 2008.

[72] A. Bonanno and M. Reuter, "Renormalization group improved black hole spacetimes," Physical Review D: Particles and Fields, vol. 62, Article ID 043008, 2000.

[73] A. Bonanno and M. Reuter, "Spacetime structure of an evaporating black hole in quantum gravity," Physical Review D: Particles and Fields, vol. 73, no. 8, Article ID 083005, 10 pages, 2006.

[74] M. Bojowald, R. Goswami, R. Maartens, and P. Singh, "Black hole mass threshold from nonsingular quantum gravitational collapse," Physical Review Letters, vol. 95, no. 9, Article ID 091302, 2005.

[75] M. Bojowald, "Nonsingular black holes and degrees of freedom in quantum gravity," Physical Review Letters, vol. 95, no. 6, Article ID 061301, 4 pages, 2005. 

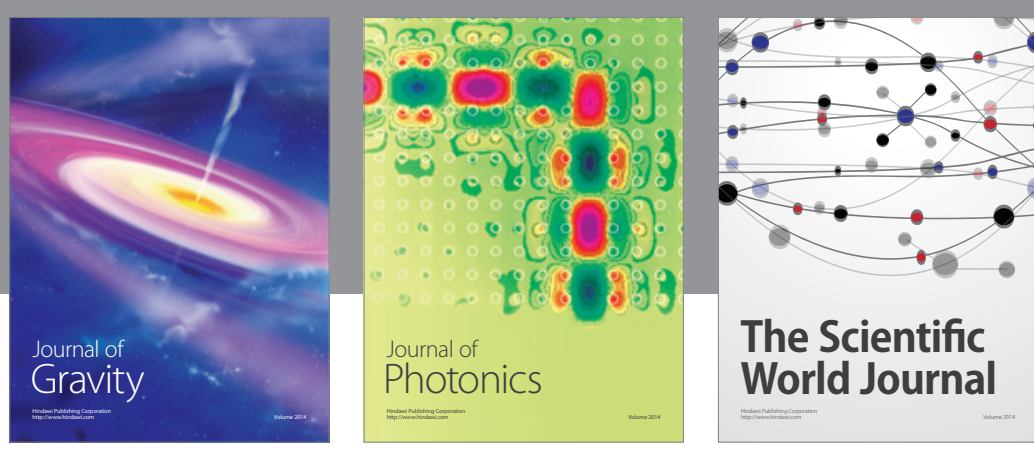

The Scientific World Journal
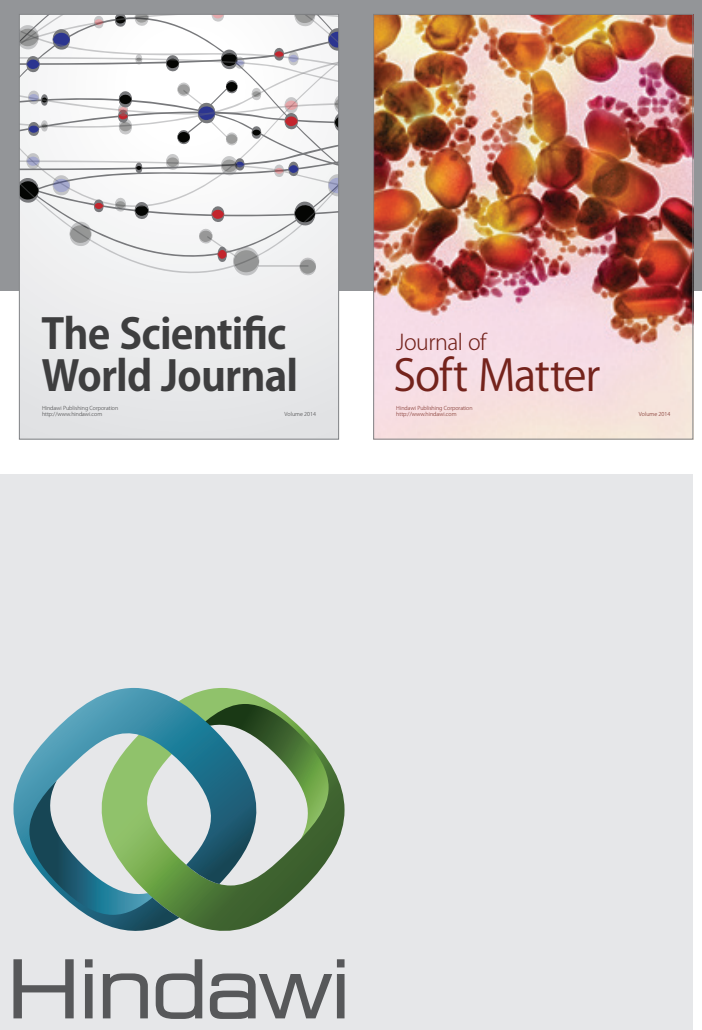

Submit your manuscripts at

http://www.hindawi.com

nternational Journal of

Statistical Mechanics
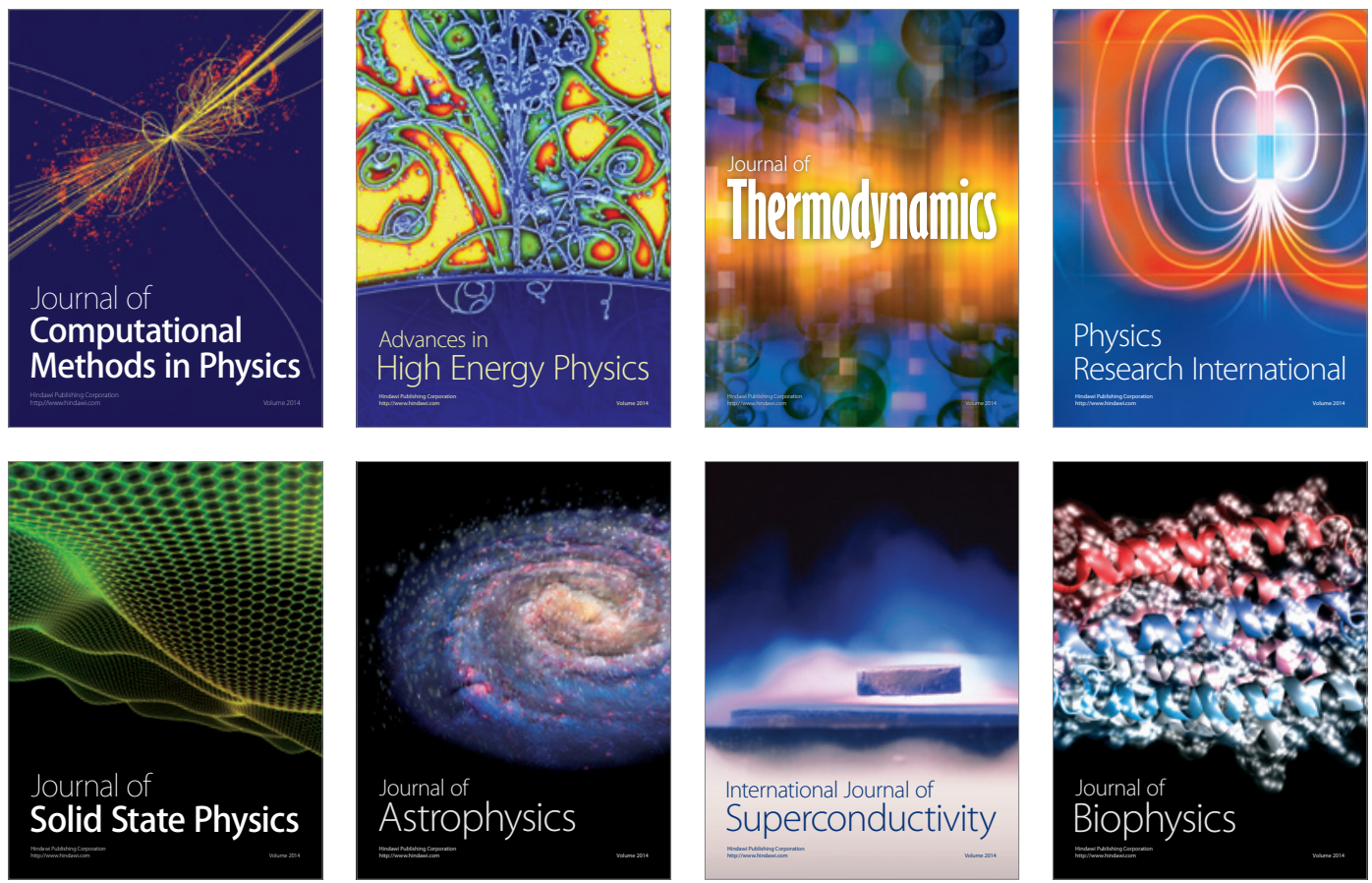
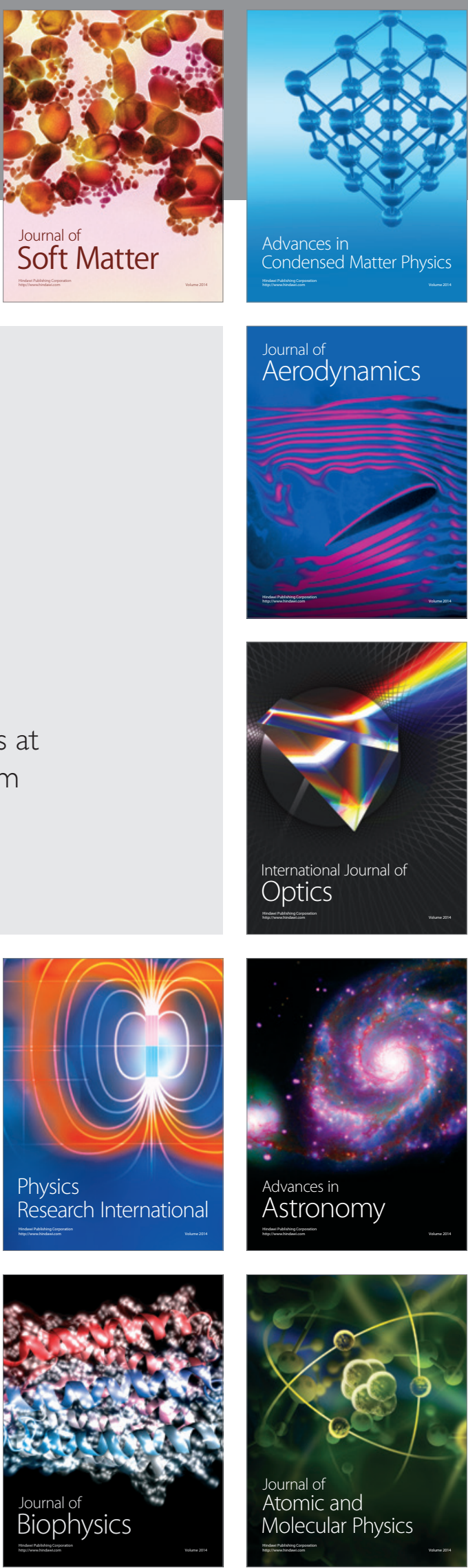